05

\title{
К статической устойчивости трубопровода
}

\author{
(C) А.Г. Хакимов \\ Институт механики им. Р.Р. Мавлютова Уфимского федерального исследовательского центра РАН, \\ 450054 Уфра, Россия \\ e-mail: hakimov@anrb.ru
}

Поступило в Редакцию 28 мая 2019 г.

В окончательной редакции 28 мая 2019 г.

Принято к публикации 21 октября 2019 г.

На основе предположения о начальной деформированной форме трубопровода без начальных напряжений определено критическое усилие сжатия. Трубопровод находился под действием внутреннего и внешнего давлений, сжимающей силы. По трубопроводу протекала жидкость с заданной плотностью. Учитывались осесимметричное расширение трубы и ее продольное укорочение, изменение температуры стенки трубы, образование арки выброса. Изучено статическое взаимодействие неустойчивостей под действием вышеуказанных факторов.

Ключевые слова: трубопровод, давление, критическое усилие сжатия.

DOI: 10.21883/JTF.2020.04.49086.214-19

\section{Введение}

Актуальность настоящей работы заключается в том, что до сих пор в литературе встречаются ошибочные трактовки действия внутреннего давления в трубопроводе на его устойчивость. Трубопроводы широко применяются в нефте-газо-продуктопроводах, в ракетных и авиационных двигателях, в воздушной и гидравлической системах самолетов. В гидроупругих системах одновременно происходит взаимодействие упругих и гидродинамических неустойчивостей. Внешние воздействия могут быть как независимыми друг от друга, так и связанными [1]. Изучению продольной устойчивости трубопроводов посвящено много работ. Отметим фундаментальные работы [2-4], в которых рассмотрены все основные случаи возможной потери трубопроводом устойчивости в минеральных грунтах, и выполнены аналитические и экспериментальные исследования в данном направлении. В перечисленных и других работах авторы несколько поразному трактуют потерю трубопроводом устойчивости, вкладывают разный смысл в данное понятие, а также с различных позиций как качественно, так и количественно поясняют факт его возможного появления [2]. Например, в [5] приведен анализ литературных источников, откуда следует, что в одних работах [6-9] уравнение продольно-поперечного изгиба трубопровода имеет вид, отличный от уравнения, приведенного в $[10,11]$. По данным ООО „Газпром трансгаз Сургут“, эксплуатирующего магистральный газопровод „Уренгой-СургутЧелябинск“, значительная протяженность трубопровода $(10 \%)$ имеет участки с непроектным положением в виде оголения, всплытия, образования арочных выбросов. В большинстве случаев вывода участков трубопровода на капитальный ремонт приходится сталкиваться именно с непроектным положением. Процесс изменения проектного положения обусловлен сложным сочетанием инженерно-гидрогеологических условий, и их прогнозирование на стадии проектирования почти невозможно. Причинами потери продольной устойчивости подземного газопровода являются: воздействие температурного перепада перекачиваемого продукта на материал трубопровода; уменьшение внутреннего давления газа (неверное утверждение - примечание автора); сезонные изменения характеристик грунта, связанные с обводнением трассы; отступление от технологии укладки трубопровода в траншею [12]. Цель настоящей работы определение критического усилия сжатия при действии различных факторов.

\section{Постановка задачи}

Примем, что упругий трубопровод „закреплен“ на защемленных скользящих „опорах“, расположенных на расстоянии $L$ друг от друга, причем „опоры“ не препятствуют движению жидкости с плотностью $\rho_{i}$ и скоростью $U_{i}$ внутри трубопровода вдоль его оси (рис. 1). Расстояние между „опорами“ будем считать длиной арки выброса трубопровода. На скользящих опорах прогиб и угол поворота равны нулю. Ускорение $G$ направлено перпендикулярно к оси трубопровода. Предполагается идеальность и несжимаемость жидкостей. Трубопровод сжимается продольной силой $P$. Сила $P$, давления внутри и вне трубопровода $p_{i}, p_{e}$ и скорость $U_{i}$ изменяются независимо друг от друга. Интенсивность их возрастания от нуля будем считать такой, чтобы инерционные силы в системе были малы.

При $P=0, U_{i}=0, p_{i}=0, p_{e}=0$ трубопровод имеет малое отклонение от оси $x$ в виде

$$
w_{0}=W_{0 n} \sin ^{2} n \beta x, \beta=\pi / L, \quad n=1,2, \ldots,
$$

где $W_{0 n}$ - амплитуда малого начального отклонения. При этом остаточные напряжения в нем отсутствуют, 


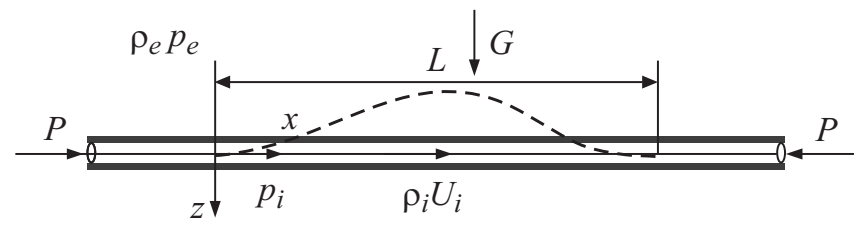

Рис. 1. Расчетная схема трубопровода.

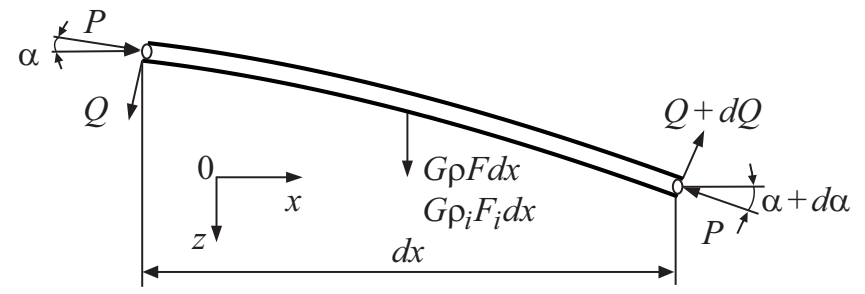

Рис. 2. Схема действия сил на упругий трубопровод при его изгибе в плоскости $z x$.

например, в результате отжига трубопровода [1]. Сумма проекций на ось $z$ всех сил, действующих на элемент длиной $d x$ (рис. 2), равна [1]

$$
\begin{aligned}
& Q \cos \alpha-(Q+d Q) \cos (\alpha+d \alpha)+P \sin \alpha \\
& -P \sin (\alpha+d \alpha)+\left[p_{i} F_{i}-p_{e}\left(F+F_{i}\right) \sin \alpha\right. \\
& -\left[p_{i} F_{i}-p_{e}\left(F+F_{i}\right)\right] \sin (\alpha+d \alpha)+G\left(\rho F+\rho_{i} F_{i}\right) d x \\
& -G \rho_{e}\left(F+F_{i}\right) d x-q d x-\rho_{i} U_{i}^{2} k d x=0,
\end{aligned}
$$

где $Q$ - перерезывающая сила, $q$ - интенсивность распределенной выталкивающей силы, $k$ - кривизна осевой линии трубопровода, $\rho, h, F-$ плотность, толщина стенки и площадь поперечного сечения трубопровода, $F_{i}$ - площадь сечения в свету трубопровода. Давления внутри и вне трубопровода определяются по формулам

$$
\begin{gathered}
p_{i}=p_{i 0}+G \rho_{i}\left(w_{0}+w\right), \\
p_{e}=p_{e 0}+G \rho_{e}\left(w_{0}+w\right), p_{e 0}=p_{0}+G \rho_{e} H .
\end{gathered}
$$

Здесь $p_{0}$ - давление на поверхности жидкости на высоте $H$ от трубопровода, $p_{i 0}, p_{e 0}$ - давления жидкости внутри и вне трубопровода на расчетной глубине. Из уравнения моментов в том же приближении следует $Q d x-d M=0$, где в выражение изгибающего момента $M=D d^{2} w / d x^{2}$ не входит $w_{0}$ ввиду указанного выше предположения об отсутствии напряжений до начала внешних воздействий. Принимая в линейной задаче $\cos \alpha=1, \sin (\alpha+d \alpha 0=\alpha+d \alpha$ и учитывая $\alpha=d\left(w_{0}+w\right) / d x, d \alpha=\left(d^{2}\left(w_{0}+w\right) / d x^{2}\right) d x$, получаем уравнение изгиба трубопровода относительно текущего прогиба $w(x)[13]$, где линеаризованное уравнение запи- сывается в следующем виде:

$$
\begin{array}{r}
D \frac{d^{4} w}{d x^{4}}+\left[P+p_{i 0} F_{i}-p_{e 0}\left(F+F_{i}\right)+\rho_{i} F_{i} U_{i}^{2}\right] \\
\times \frac{d^{2}\left(w_{0}+w\right)}{d x^{2}}=0, \quad D=E J, F_{i}=\pi R_{i}^{2}, \\
F=\pi\left[\left(R_{i}+h\right)^{2}-R_{i}^{2}\right], \quad J=\pi\left[\left(R_{i}+h\right)^{4}-R_{i}^{4}\right] / 4,
\end{array}
$$

где $E, R_{i}$ - модуль упругости, внутренний радиус поперечного сечения трубопровода.

\section{Изгиб трубопровода}

Примем частное решение уравнения (2) в виде

$$
w=W_{n} \sin ^{2} n \beta x, \quad n=1,2, \ldots,
$$

где $W_{n}$ - амплитуда малого отклонения. Подставляя (1), (3) в уравнение (2), получаем отношение амплитуды текущего прогиба к амплитуде начального прогиба в виде [13]

$$
\begin{aligned}
& \frac{W_{n}}{W_{0 n}}=\frac{R_{n}}{P_{E}\left(n^{2} \beta\right)^{2}-R_{n}}, \quad P_{E}=4 D \beta^{2}=\frac{4 \pi^{2} E J}{L^{2}} \\
& R_{n}=\left[P+p_{i 0} F_{i}-p_{e 0}\left(F+F_{i}\right)+\rho_{i} F_{i} U_{i}^{2}\right](n \beta)^{2}
\end{aligned}
$$

Здесь $P_{E}$ - критическое значение статической продольной сжимающей силы на трубопровод $(n=1)$. Из (4) видно, что прогиб тем больше, чем больше величина осевого усилия сжатия трубопровода $P$, давление внутри трубопровода $p_{i 0}$, скоростной напор внутри трубопровода $\rho_{i} U_{i}^{2}$ и меньше критическое значение статической продольной сжимающей силы на трубопровод $P_{E}$, давление вне трубопровода $p_{e 0}$. При $R_{n}=P_{E}\left(n^{2} \beta\right)^{2}$ прогиб в рассматриваемом линейном приближении растет неограниченно. Здесь $R_{n}$ представляет собой критическую комбинацию внешних воздействий $P, p_{i 0}, p_{e 0}$, $\rho_{i} U_{i}^{2}$ для системы с заданными параметрами материалов и размерами $E, \rho_{i}, h, L, R_{i}$. С возрастанием внешних воздействий рост прогиба (4) происходит наиболее быстро при $n=1$. Указанная критическая комбинация внешних воздействий по (4) является минимальной при $n=1$. Под действием перепада давления $p^{*}=p_{i 0}-p_{e 0}$ происходит осесимметричное расширение трубы и ее продольное укорочение, что в зависимости от коэффициента Пуассона материала и условий закрепления трубы на опорах в продольном направлении приводит к образованию растягивающей продольной силы $\left(p_{i 0}-p_{e 0}\right) F_{i 0} \chi$ и соответственно распределенной поперечной силы $\left(p_{i 0}-p_{e 0}\right) F_{i 0} \chi k$, направленной в сторону вогнутости осевой линии. Величина $\chi$ включает в себя указанные выше параметры. 


\section{Осесимметричная деформация трубопровода}

Для определения величины $\chi$ нужно рассмотреть осесимметричную деформацию под действием перепада давления $p^{*}=p_{i 0}-p_{e 0}$. При пренебрежении инерционностью окружная сила равна $N_{* \theta}=p_{*} R$. Из закона Гука следует [14]

$$
\begin{aligned}
& N_{* \theta}=\frac{E h}{1-v^{2}}\left[\varepsilon_{\theta *}+v \varepsilon_{*}-(1+v) \alpha T\right], \\
& N_{*}=\frac{E h}{1-v^{2}}\left[\varepsilon_{*}+v \varepsilon_{\theta *}-(1+v) \alpha T\right],
\end{aligned}
$$

где $N_{* \theta}$ и продольная сила $N_{*}$ - принимаются приходящимися на единицу длины, $v-$ коэффициент Пуассона, $\alpha$ - коэффициент линейного теплового расширения, $T$ - изменение температуры трубопровода, $\varepsilon_{\theta *}, \varepsilon_{*}-$ деформации. Исключив $\varepsilon_{\theta *}$ из (5), получаем

$$
N_{*}=\left(p_{i 0}-p_{e 0}\right) R_{i} v+E h\left(\varepsilon_{*}-\alpha T\right) .
$$

Продольная деформация постоянна по длине пролета $\varepsilon_{*}=A$ или

$$
\begin{gathered}
\varepsilon_{*}=\frac{\partial u_{*}}{\partial x}+\frac{1}{2}\left(\frac{\partial w_{*}}{\partial x}\right)^{2}=A, \\
u_{*}=A x+B-\frac{1}{2} \int_{0}^{x}\left(\frac{\partial w_{*}}{\partial x}\right)^{2} d x .
\end{gathered}
$$

Константы $A$ и $B$ определяем из условий $N_{*}=C u_{*}$ $(x=0), u_{*}=0(x=L)$, где $C-$ продольная жесткость опоры единичной дуги. В соответствии с выражениями (6), (7) полное осевое усилие по трубе равно

$$
\begin{gathered}
2 \pi R_{i} N_{*}=\left(p_{i 0}-p_{e 0}\right) F_{i} \chi-\beta T+\gamma W_{n}^{2}, \\
\chi=\frac{2 \nu}{1+\lambda}, \lambda=\frac{E h}{C L}, \beta=\frac{2 \pi \alpha E h R_{i}}{(1+\lambda)}, \gamma=\frac{\pi^{3} E h R_{i}}{2(1+\lambda) L^{2}} .
\end{gathered}
$$

Коэффициент $\lambda$ представляет собой отношение жесткостей трубы и опоры в продольном направлении. При малом отношении этих жесткостей $\chi \rightarrow 2 v$, при большом их отношении $\chi \rightarrow 0$. Если осевое усилие сжатия трубопровода $P=P_{0}$ при $p_{i 0}=0, p_{e 0}=0, \rho_{i} U_{i}^{2}=0$, $T=0$, то справедливо равенство

$$
P=P_{0}-2 \pi R_{i} N_{*}=P_{0}-\left(p_{i 0}-p_{e 0}\right) F_{i} \chi+\beta T-\gamma W_{n}^{2} .
$$

Из выражения (4) с учетом соотношения (8) получаем критическое значение сжимающей трубопровод силы $P_{0}$ при $n=1$

$$
\begin{gathered}
P_{0 c r}=P_{E} n^{2}+\left(p_{i 0}-p_{e 0}\right) F_{i} \chi-p_{i 0} F_{i}+p_{e 0}\left(F+F_{i}\right) \\
-\rho_{i} F_{i} U_{i}^{2}-\beta T+\gamma W_{n}^{2},
\end{gathered}
$$

или

$$
\begin{gathered}
P_{0 c r}=P_{E} n^{2}-p_{i 0} F_{i}(1-\chi)+p_{e 0} F+p_{e 0} F_{i}(1-\chi) \\
-\rho_{i} F_{i} U_{i}^{2}-\beta T+\gamma W_{n}^{2},
\end{gathered}
$$

которое представляет собой обобщение классического критического значения в задаче Эйлера за счет учета давлений внутри и вне трубопровода $p_{i 0}, p_{e 0}$, скоростного напора внутри трубопровода $\rho_{i} U_{i}^{2}$, температуры стенки трубопровода $T$, максимального прогиба трубопровода $W_{n}$ при образовании арки выброса. Из (9) видно, что критическое значение сжимающей трубопровод силы $P_{0}$ тем больше, чем больше критическое значение статической продольной сжимающей силы Эйлера на трубопровод $P_{E}$, давление вне трубопровода $p_{e 0}$ и меньше давление внутри трубопровода $p_{i 0}$, скоростной напор внутри трубопровода $\rho_{i} U_{i}^{2}$, температура стенки трубопровода $T$. Также отметим, что с увеличением максимального прогиба трубопровода $W_{n}$ происходит увеличение критического значения сжимающей трубопровод силы $P_{0}$. Первое слагаемое в правой части выражения (9) представляет критическую осевую сжимающую силу Эйлера, второе слагаемое возникает при осесимметричном расширении трубы и ее продольном укорочении под действием перепада давления $p^{*}=p_{i 0}-p_{e 0}$, что в зависимости от коэффициента Пуассона материала и условий закрепления трубы на опорах приводит к образованию растягивающей продольной силы, третье слагаемое, умноженное на кривизну $k$, представляет поперечную распределенную силу, направленную в сторону выпуклости осевой линии, четвертое слагаемое, умноженное на кривизну $k$, представляет поперечную распределенную силу, направленную в сторону вогнутости осевой линии при изгибе трубы под действием внешнего давления $p_{e 0}$, пятое слагаемое, умноженное на кривизну $k$, представляет поперечную распределенную силу, направленную в сторону выпуклости осевой линии при изгибе трубы под действием скоростного напора $\rho_{i} U_{i}^{2}$, шестое слагаемое - это сжимающая сила, возникающая при увеличении температуры стенки трубопровода $T$ и учитывающая условия закрепления трубы на опорах, седьмое слагаемое - это растягивающая сила при образовании арки выброса, учитывающая условия закрепления трубы на опорах. Частные случаи рассмотрены в $[13,15]$.

\section{Анализ результатов и примеры}

Расчеты проведены для следующих параметров трубы: модуль упругости материала трубопровода $E=2.0 \cdot 10^{11} \mathrm{~N} / \mathrm{m}^{2}$, плотность $\rho=7800 \mathrm{~kg} / \mathrm{m}^{3}$, коэффициент Пуассона $v=0.3$, коэффициент линейного теплового расширения $\alpha=11.3 \cdot 10^{-6} \mathrm{deg}^{-1}$, внутренний радиус поперечного сечения трубопровода $R_{i}=0.496 \mathrm{~m}$, толщина стенки $h=14 \mathrm{~mm}$, температура стенки $T=5^{\circ} \mathrm{C}$, давление газа внутри трубопровода 
$p_{i 0}=5 \mathrm{MPa}$, плотность газа $\rho_{i}=100 \mathrm{~kg} / \mathrm{m}^{3}$, скорость течения газа $U_{i}=10 \mathrm{~m} / \mathrm{s}$, расстояние между опорами $L=120 \mathrm{~m}$, продольная жесткость опоры единичной дуги $C=10^{8} \mathrm{~N} / \mathrm{m}^{2}$, давление вне трубопровода $p_{e 0}=0 \mathrm{MPa}$, амплитуда малого отклонения $W_{n}=0.1 \mathrm{~m}$. Расчеты по формуле (10) дают: критическое значение сжимающей трубопровод силы $P_{0 c r}=0.106 \mathrm{MN}$, критическая сжимающая сила Эйлера равна $3.069 \mathrm{MN}$. Критическое значение сжимающей трубопровод силы уменьшается на $2.567 \mathrm{MN}$ под действием внутреннего давления, на $0.007 \mathrm{MN}$ вследствие движения газа по трубопроводу, на $0.399 \mathrm{MN}$ из-за увеличения температуры стенки трубы и увеличивается на $0.012 \mathrm{MN}$ за счет появления арки выброса с амплитудой $W_{n}=0.1 \mathrm{~m}$.

Арки выброса можно рассматривать как компенсаторы осевых усилий и в некоторых случаях их предусматривать в проектах новых трубопроводов. Так как максимальное возможное значение $\chi=2 v$, из выражения (9) следует, что внутреннее давление в трубопроводе приводит к снижению критического значения сжимающей трубопровод силы. В статье [12] во введении отмечено, что причиной потери продольной устойчивости подземного трубопровода является уменьшение внутреннего давления газа, что противоречит формуле (9). К снижению критического значения сжимающей трубопровод силы приводит также движение жидкости по трубопроводу. Из выражения (9) следует, что критическая сжимающая сила $P_{0 c r}$ может быть отрицательной или трубопровод может потерять устойчивость под действием растягивающих осевых усилий. Видно, что увеличение внутреннего давления $p_{i 0}$, скоростного напора внутри трубопровода $\rho_{i} U_{i}^{2}$ и температуры его стенки, уменьшение изгибной жесткости $E J$ трубопровода, внешнего давления $p_{e 0}$ приводят к уменьшению критического значения силы $P_{0}$. Для исследования вклада каждого слагаемого в выражении (9) запишем его в виде

$$
\begin{gathered}
P_{0 c r}=P_{E}+P_{P i}+P_{P e}+P_{U i}+P_{T}+P_{W n}, P_{E}=P_{E} n^{2}, \\
P_{P i}=-p_{i 0} F_{i}(1-\chi), P_{P e}=p_{e 0} F+p_{e 0} F_{i}(1-\chi), \\
P_{U i}=-\rho_{i} F_{i} U_{i}^{2}, P_{T}=-\beta T, P_{W n}=\gamma W_{n}^{2} .
\end{gathered}
$$

На рис. 3 приводятся зависимости критического значения сжимающей трубопровод силы $P_{0 c r}$ от давления внутри трубопровода $p_{i 0}$ для давления вне трубопровода $p_{e 0}=0 \mathrm{MPa}$ (кривая 1), $p_{e 0}=0.5 \mathrm{MPa}$ (кривая 2), $p_{e 0}=1.0 \mathrm{MPa}$ (кривая 3) при вышеприведенных данных и температуре стенки $T=1^{\circ} \mathrm{C}$.

Видно, что с увеличением внутреннего давления в трубопроводе происходит уменьшение критического значения сжимающей трубопровод силы. Можно отметить, что с увеличением внешнего давления происходит увеличение критического значения сжимающей трубопровод силы.

На рис. 4 приводятся зависимости $P_{P i}$ от жесткости опоры единичной дуги $C$ для давления внутри трубопровода $\left.p_{i 0}=6 \mathrm{MPa} \mathrm{(кривая} 1\right), p_{i 0}=8 \mathrm{MPa} \mathrm{(кривая} \mathrm{2),}$

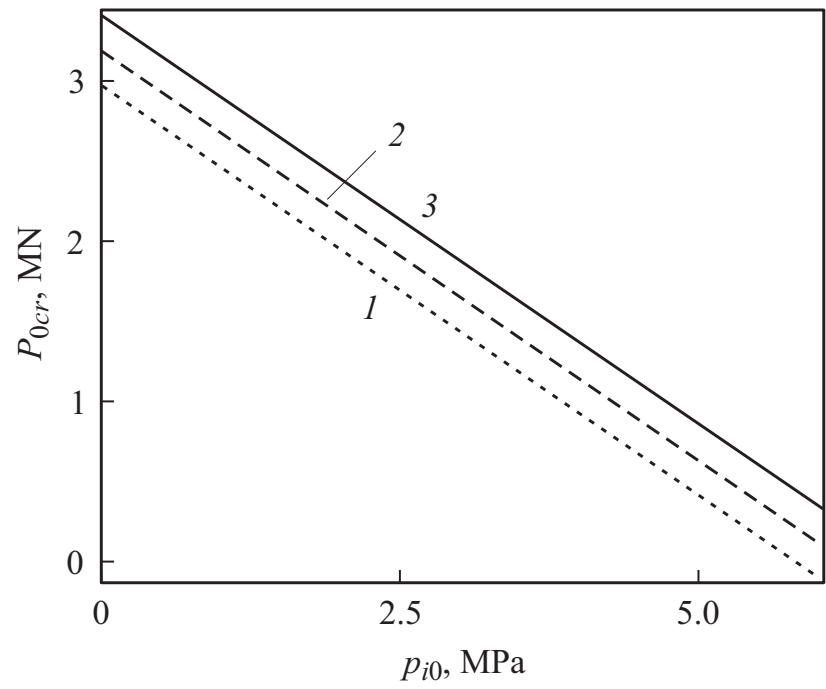

Рис. 3. Зависимости критического значения сжимающей трубопровод силы $P_{0 c r}$ от давления внутри трубопровода $p_{i 0}$.

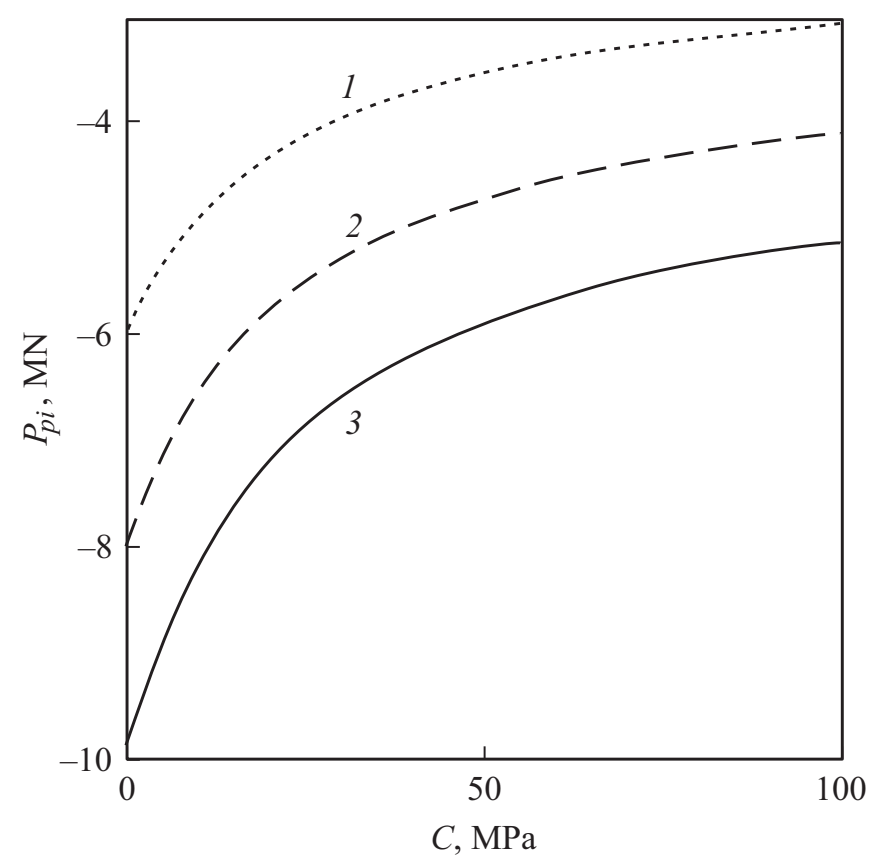

Рис. 4. Зависимости $P_{P i}$ от жесткости опоры единичной дуги $C$.

$p_{i 0}=10 \mathrm{MPa}$ (кривая 3) при вышеприведенных в примеpe данных. Видно, что с увеличением жесткости опоры единичной дуги $C$ происходит уменьшение влияния внутреннего давления на $P_{P i}$.

На рис. 5 приводятся зависимости $P_{T}$ от жесткости опоры единичной дуги $C$ для температуры стенки трубопровода $T=50^{\circ} \mathrm{C}$ (кривая 1), $T=60^{\circ} \mathrm{C}$ (кривая 2), $T=70^{\circ} \mathrm{C}$ (кривая 3) при вышеприведенных в примере данных. Видно, что с увеличением жесткости опоры единичной дуги $C$ происходит увеличение влияния температуры стенки трубопровода на $P_{T}$. 


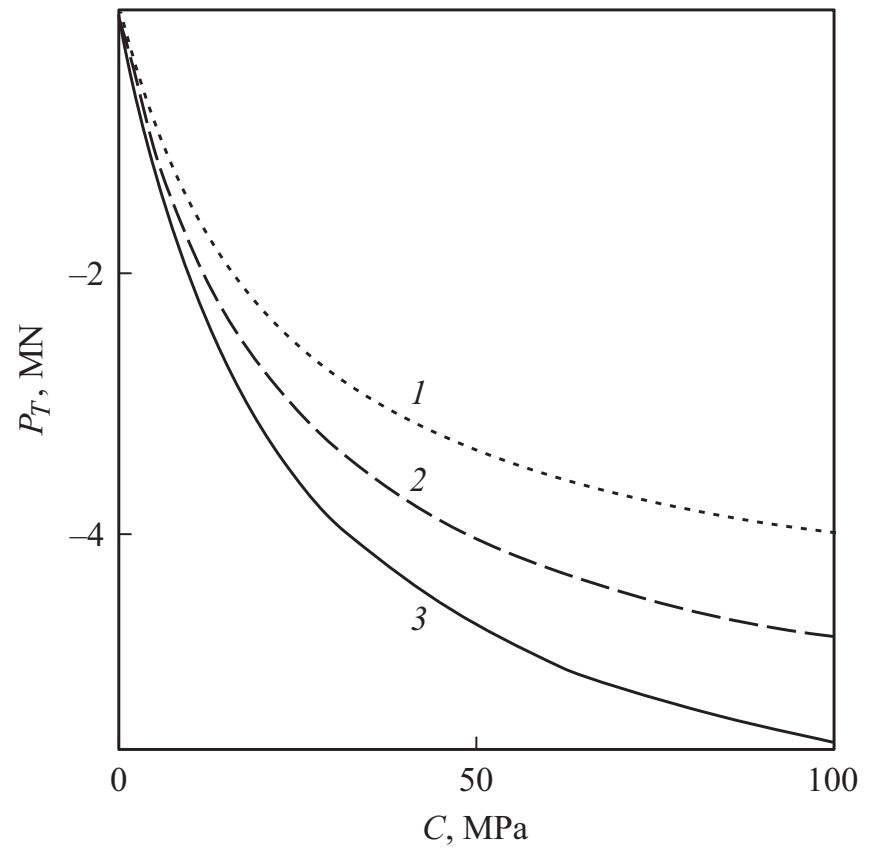

Pис. 5. Зависимости $P_{T}$ от жесткости опоры единичной дуги $C$.

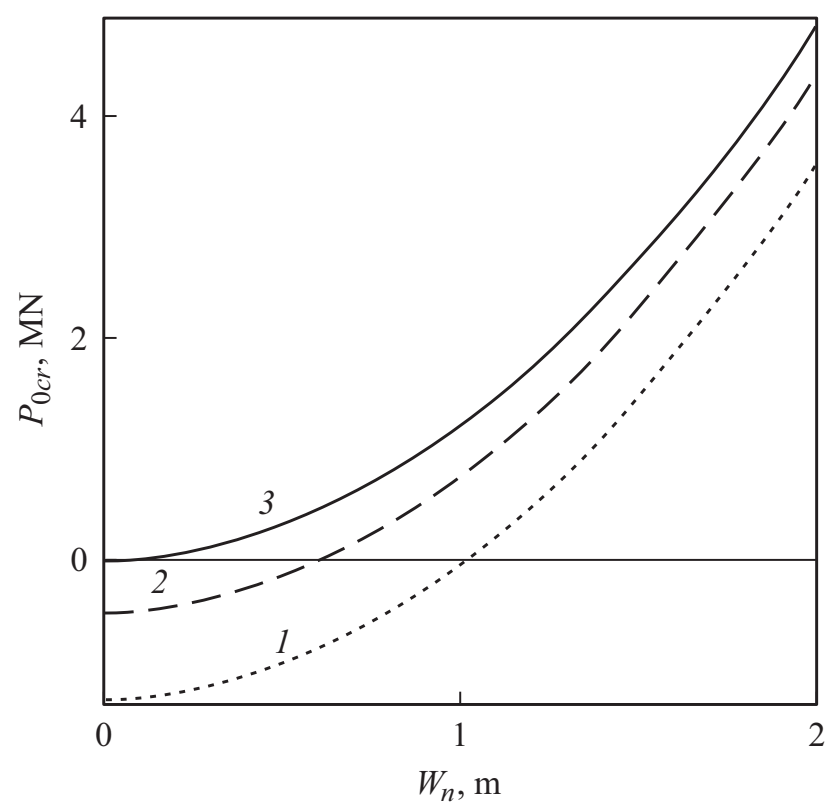

Рис. 6. Зависимости критического значения сжимающей трубопровод силы $P_{0 c r}$ от максимального прогиба трубопровода $W_{n}$.

На рис. 6 приводятся зависимости критического значения сжимающей трубопровод силы $P_{0 c r}$ от максимального прогиба трубопровода $W_{n}$ для жесткости опоры единичной дуги $C=20 \mathrm{MPa}$ (кривая 1$), 50 \mathrm{MPa}$ (кривая 2), $100 \mathrm{MPa} \mathrm{(кривая} \mathrm{3).} \mathrm{С} \mathrm{увеличением} \mathrm{максимального} \mathrm{про-}$ гиба трубопровода происходит увеличение критического значения сжимающей трубопровод силы.
Если образовалась арка выброса, то можно определить критическое значение сжимающей трубопровод силы $P_{0 c r}$. Если действующий трубопровод имеет арку выброса и из эксперимента известно перемещение трубопровода в начале координат $(\mathrm{x}=0)$, которое равно $B[15]$, то продольная жесткость опоры единичной дуги $C$ определяется по формуле

$$
C=\frac{-4\left(B L+\alpha T L^{2}-\pi^{2} W_{n}^{2} / 4\right) E h+p_{i 0} R_{i} \nu L^{2}}{B L^{2}} .
$$

Далее по известным остальным параметрам можно определить действующее критическое усилие по формуле (9).

\section{Выводы}

С увеличением внутреннего давления в трубопроводе происходит уменьшение критического значения сжимающей трубопровод силы, а с увеличением внешнего давления происходит увеличение критического значения сжимающей трубопровод силы. С увеличением жесткости опоры единичной дуги происходит увеличение влияния температуры стенки трубопровода на сжимающую трубопровод силу. С увеличением максимального прогиба трубопровода происходит увеличение критического значения сжимающей трубопровод силы. Получена формула для определения продольной жесткости опоры единичной дуги по известному из эксперимента перемещению трубопровода в начале координат. Полученные результаты позволяют анализировать устойчивость трубопроводных систем. Результаты работы могут найти применение для анализа статической устойчивости трубопроводов на этапе проектирования, эксплуатации и ремонта арок выброса. Получено критическое значение сжимающей трубопровод силы $P_{0}$, которое представляет собой обобщение классического критического значения сжимающей трубопровод силы в задаче Эйлера за счет учета давлений внутри и вне трубопровода, скоростного напора внутри трубопровода, температуры стенки трубопровода, максимального прогиба трубопровода при образовании арки выброса.

\section{Благодарности}

Автор выражает благодарность член-корреспонденту РАН М.А. Ильгамову за обсуждение результатов работы.

\section{Финансирование работы}

Работа поддержана средствами государственного бюджета по государственному заданию на 2019-2022 г. (№ 0246-2019-0088) и грантом РФФИ (№ 18-01-00150).

\section{Конфликт интересов}

Автор заявляет, что у него нет конфликта интересов. 


\section{Список литературы}

[1] Ильгамов М.А. // ЖТФ. 2018. Т. 63. Вып. 2. С. 163-167. DOI: 10.21883/JTF.2018.02.45401.2144

[2] Димов Л.А., Богушевская Е.М. Магистральные трубопроводы в условиях болот и обводненной местности. М.: Горная книга: Изд-во Московского гос. горного ун-та, 2010. $391 \mathrm{c}$

[3] Ясин Э.М., Черникин В.И. Устойчивость подземных трубопроводов. М.: Недра, 1968. 120 с.

[4] Айнбиндер А.Б., Камерштейн А.Г. Расчет магистральных трубопроводов на прочность и устойчивость. М.: Недра, 1982. $343 \mathrm{c}$.

[5] Гумеров К.М., Сильвестров С.А. // Проблемы сбора, подготовки и транспорта нефти и нефтепродуктов. 2017. Вып. 1 (107). C. 60-68. DOI: http://dx.doi.org/10.17122/ntjoil-2017-1-60-68

[6] Бабин Л.А., Быков Л.И., Волохов В.Я. Типовые расчеты по сооружению трубопроводов. М.: Недра, 1979. 176 с.

[7] Бородавкин П.П., Березин В.Л. Сооружение магистральных трубопроводов. М.: Недра, 1977. 407 с.

[8] Мугаллимов Ф.М., Багманов Р.Р., Гумеров А.К., Мугаллимов И.Ф. // Проблемы сбора, подготовки и транспорта нефти и нефтепродуктов. 2015. Вып. 3 (101). С. 88-96.

[9] Чучкалов М.В., Гумеров К.М. // Транспорт и хранение нефтепродуктов и углеводородного сырья. 2014. № 2. C. 3-6.

[10] Коробков Г.Е., Зарипов Р.М., Шаммазов И.А. Численное моделирование напряженно-деформированного состояния и устойчивости трубопроводов и резервуаров в осложненных условиях. СПб.: Недра, 2009. 410 с.

[11] Петров И.П., Спиридонов В.В. Надземная прокладка трубопроводов. М.: Недра, 1973. 472 с.

[12] Сысоев Ю.С. // Изв. вузов. Нефть и газ. 2012. № 1. С. $72-$ 76.

[13] Хакимов А.Г. // Вестник Пермского нац. исследовательского политех. ун-та. Механика. 2018. № 3. С. 87-94. DOI: $10.15593 /$ perm.mech/2018.3.09

[14] Коваленко А.Д. Основы термоупругости. Киев: Наук. думка, 1970. $307 \mathrm{c}$.

[15] Хакимов А.Г. // Экспозиция. Нефть. Газ. 2019. № 1 (68). C. $48-52$. https://elibrary.ru/item.asp?id $=37028571$ 